\title{
Gastrodin protects dopaminergic neurons via insulin-like pathway in a Parkinson's disease model
}

\author{
Jinyuan Yan ${ }^{1+}$, Zhongshan Yang ${ }^{2+}$, Ninghui Zhao ${ }^{1}$, Zhiwei Li ${ }^{1}$ and Xia Cao ${ }^{1 *}$ (])
}

\begin{abstract}
Background: Recently, the use of traditional Chinese medicine (TCM) has become more generally accepted, including by the Food and Drug Administration. To expand the use of TCM worldwide, it is important to study the molecular mechanisms by which TCM and its active ingredients produce effects. Gastrodin is an active ingredient from Gastrodia elata Blume. It is reported that gastrodin has neuroprotective function in Parkinson's disease. But its mechanisms of neuroprotection remain not clear in PD. Here, we build two C. elegans PD model using 6-OHDA and transgenic animal to observe the changes of PD worms treated with or without gastrodin to confirm the function of gastrodin, then utilize mutant worms to investigate DAF-2/DAF-16 signaling pathway, and finally verify the mechanism of gastrodin in PD.

Results: Gastrodin attenuates the accumulation of a-synuclein and the injury of dopaminergic neurons, improves chemotaxis behavior in Parkinson's disease models, then recovers chemotaxis behavior by insulin-like pathway. DAF-2/DAF-16 is required for neuroprotective effect of dopamine neuron in PD.

Conclusions: Our study demonstrated that gastrodin rescued dopaminergic neurons and reduced accumulation of a-synuclein protein, and the activity of gastrodin against Parkinson's disease depended on the insulin-like DAF-2/DAF16 signaling pathway. Our findings revealed that this insulin-like pathway mediates neuroprotection of gastrodin in a Parkinson's disease model.
\end{abstract}

Keywords: Gastrodin, Dopamine neurons, a-Synuclein, DAF-2/DAF-16 pathway, Parkinson's disease

\section{Background}

The incidence and prevalence of Parkinson's disease (PD) continue to increase worldwide [1, 2]. Parkinson's disease is a common disease in older people, and is the second main neurodegenerative disease worldwide. The symptoms of PD include bradykinesia, rigidity, abnormal posture, resting tremors, olfactory dysfunction, and cognitive disorder. PD is characterized by a loss of $80 \%$ of substantia nigra dopamine (DA) neurons and the excess accumulation of $\alpha$-synuclein protein [3, 4]. Genetic

\footnotetext{
*Correspondence: 914616613@qq.com

†Jinyuan Yan and Zhongshan Yang contributed equally to this work

${ }^{1}$ The Second Affiliated Hospital of Kunming Medical University, 374 Dian Mian Road, Kunming 650101, Yunnan, China

Full list of author information is available at the end of the article
}

factors, environmental factors, aging, toxin, infection, and potentially other factors may cause PD. About 10\% of PD cases are inherited forms of disease, and many PD-associated genes have been identified, including SNCA(PARK1/4), Parkin(PARK2), LRRK2(PARK8), PINK1(PARK6), UCHL-1(PARK5), DJ-1(PARK7), ATXN2, ATXN3, PLA2G6, ATP13A2(PARK9) and so on $[5,6]$. Currently, levodopa is widely used clinically, countering PD symptoms by maintaining normal dopamine metabolism. However, levodopa only alleviates symptoms, and does not alter the development of PD. Additionally, the long-term use of levodopa may cause side-effects, such as involuntary movements, agitation, clumsiness, low blood pressure, slowed gastrointestinal peristalsis, and even mental symptoms [7]. Overall, there remains a lack of effective drugs and treatments to both 
treat symptoms and delay the onset of disease. Hence, finding a medicine with neuroprotective effects would be highly valuable for improved treatment of PD.

Gastrodin is an active ingredient of the orchid plant Gastrodia elata Blume, and is a commonly used traditional Chinese medicine. Gastrodin is reported to have antioxidant, anticonvulsant, anti-inflammatory, analgesic, sedative, and anxiolytic properties, and can improve learning and memory [8-12]. Gastrodin reduces mRNA and protein levels of inflammatory enzymes and proinflammatory cytokines that are induced by the suppression of NF-kB signalling and the phosphorylation of mitogen-activated protein kinases (MAPK) in microglial cells induced by lipopolysaccharide [9]. Gastrodin can cross the blood-brain barrier, and then is decomposed into p-hydroxy benzyl alcohol in the central nervous system, blood, and liver to protect the nervous system from damage [13]. In 6-OHDA rat model of PD, gastrodin with microinjection in intra-cerebro ventricular lowers myeloperoxidase, the levels of lipid peroxidation and NO production, rescues antioxidant capacity levels, and ameliorates motor incoordination of rats [14]. In $\mathrm{MPP}^{+}$ induced SH-SY5Y model of PD, gastrodin combination with isorhynchophylline regulates ERK1/2 and GSK-3 $\beta$ pathways, increases Nrf2 nuclear accumulation to decrease oxidative stress for neuroprotective effect [15]. Kumar et al. reports that gastrodin has an anti-apoptosis role in PD model [16]. The study of cellular PD models also suggest that gastrodin counteract oxidative stress and p38 MAPK/Nrf2 pathway to protect dopamine neurons [17]. Although gastrodin is a potential drug for the treatment of neurodegenerative diseases, its mechanisms of neuroprotection remain unclear. Hence, the pathway through which gastrodin mediates neuroprotective effect in PD should be elucidated to better understand its function and potentially expand its use for disease treatment.

In our study, we selected the Caenorhabditis elegans ( $C$. elegans) model to study PD. C. elegans is a robust animal model with many advantages because it is of small size, has a short life cycle, is inexpensive, and multiple transgenic strains exist, enabling the study of various human diseases. Here, we demonstrated that gastrodin alleviated dopamine neuron injury and $\alpha$-synuclein protein aggregation, and regulated the DAF-2/DAF-16 insulinlike signaling pathway to decrease dopaminergic neurons degeneration in a C. elegans model of Parkinson disease.

\section{Results}

Gastrodin attenuates the accumulation of a-synuclein and the injury of dopaminergic neurons in different Parkinson models

The function of gastrodin in the treatment of neurodegenerative disease has been widely reported, but its mechanism of action is poorly clarified. To determine whether gastrodin affected the normal physiological function of animals, we chose the $C$. elegans PD model. We first investigated the food clearance and pumping rate (ingestion rate) assay in wild type animals fed $0,25,50,100$, or $200 \mu \mathrm{M}$ gastrodin. The food clearance and pumping rate assay was supplied in this section for detecting the effect of gastrodin on $C$. elegans physiology and monitoring the right concentration [18-20]. As shown in Fig. 1b, the food clearance of animals with $200 \mu \mathrm{M}$ gastrodin was significantly reduced than that of worms in the absence of gastrodin from 2 to 6 days $(P<0.001, F=58.87 . d f=4$, two-way RM ANOVA following by Bonferroni post-tests). We also observed a lower pumping rate for animals fed $200 \mu \mathrm{M}$ gastrodin compared to no gastrodin group $(P=0.0077<0.01, F=3.96$, $d f=4,45)$ (Fig. 1c). Hence, we used concentrations of 25 , 50 , and $100 \mu \mathrm{M}$ gastrodin for the following assays.

We next evaluated the effect of gastrodin $(25,50$, and $100 \mu \mathrm{M})$ on two Parkinson models, one produced by 6-OHDA treatment and one produced by transgenic worm overexpression of $\alpha$-synuclein. After 6-OHDA treatment, dopamine neurons and axons marked with BZ555 [egIs1 [dat-1p::GFP]] were impaired (Fig. 2a). However, the expression of Pdat-1::gfp in the 6-OHDA induced model with addition of 50 and $100 \mu \mathrm{M}$ gastrodin showed obviously higher than that of 6-OHDA PD model $(P=0.0030,0.0067<0.01 ; F=1.592$. 2.719; $d f=6$, 6; Fig. 2a, b). A lesser effect was seen for higher amounts of $25 \mu \mathrm{M}(P=0.0940>0.05, F=2.880, d f=6)$, suggesting $50 \mu \mathrm{M}$ gastrodin was required for better protection in the Parkinson model. At $50 \mu \mathrm{M}$ gastrodin in OP50/NGM plates, the accumulation of $\alpha$-synuclein in transgenic strain OW13 [grk-1(ok1239) X; pkIs2386 IV] overexpressing the human $\alpha$-synuclein protein showed a remarkable decrease $(P=0.0086<0.01, F=4.49, d f=4$; Fig. 2c, d). Taken together, these data suggest that the addition of $50 \mu \mathrm{M}$ gastrodin had an obvious effect on two parameters of PD disease.

\section{Gastrodin improves chemotaxis in PD animals}

The loss of dopamine neurons can affect olfactory transmissions and olfactory bulb function [21-24]. About $90 \%$ of patients with Parkinson's disease exhibit olfactory dysfunction symptoms [24]. Aging, $\alpha$-synuclein accumulation, inflammation response, tau deposition, dopamine loss, and environmental elements may contribute to olfactory deficits in PD [24]. Hence, olfactory dysfunction may serve as a sensitive marker of PD [25]. Thus, we measured olfactory function in the PD model by observing the attractive behavior/chemotaxis behavior to the volatile compounds 2-heptanone and benzaldehyde. A significant decrease in chemotaxis to 2-heptanone in 

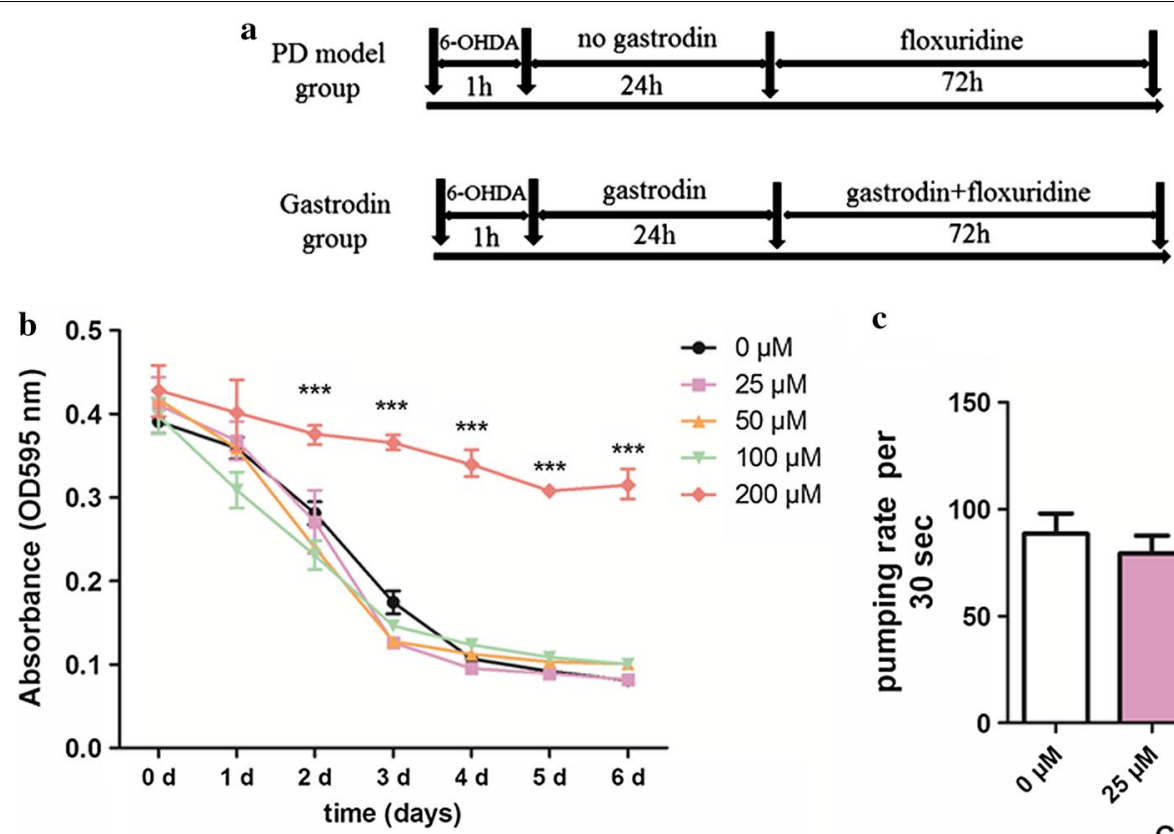

c

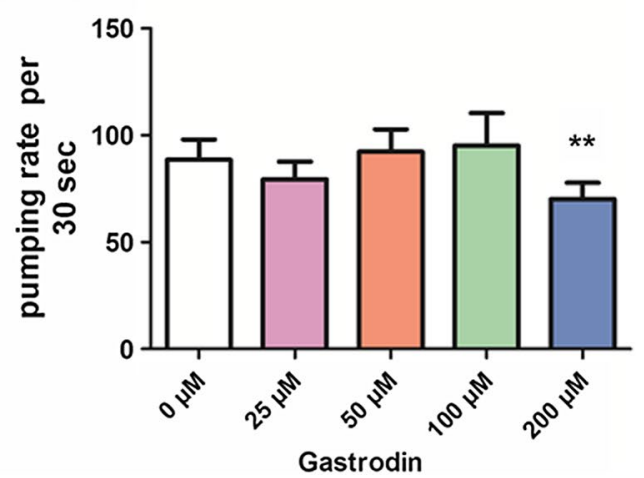

Fig. 1 Determination of the appropriate assay concentration of gastrodin. a The schematic diagram of the timeline about building PD model and gastrodin treatment. $\mathbf{b}$ The food clearance rates of worms for different concentrations of gastrodin were determined. The statistical differences were analyzed using two-way RM ANOVA following by Bonferroni post-tests to compare replicate means by row (c) Gastrodin at $200 \mu \mathrm{M}$ obviously reduced the pumping rates of worms. The statistical differences were analyzed using one-way ANOVA. ns $P \geq 0.05,{ }^{*} P<0.05$; ${ }^{* *} P<0.01$; ${ }^{* * *} P<0.001$

both 6-OHDA-treated and OW13 worms was observed relative to the chemotaxis behavior of wild type worms $(P=0.0041, \quad 0.0073<0.01 ; \quad F=5.384,1.420 ; \quad d f=4,4$; Fig. 3a), but treatment with gastrodin increased the chemotaxis index for the deficient worms $(P=0.0215$, $0.0263<0.05 ; F=2.566,2.401 ; d f=4$, 4; Fig. 3b). This result indicated that chemotaxis can be used as an indicator of PD in further experiments. In addition, expression of BZ555, as indicated by GFP signal, was not changed after treatment with gastrodin relative to the control $(P=0.9798>0.05, F=1.167, d f=6$; Additional file 1: Fig. S1). Overall, we conclude that 6-OHDA-treated and OW13 transgenic worms show deficient olfactory function as evidenced by altered chemotaxis behavior to volatiles.

\section{Gastrodin recovers chemotaxis behavior by insulin-like pathway}

Accumulating evidences suggest that insulin-like pathway may be involved in neurodegenerative diseases [26-28]. In C. elegans, insulin-like signaling mainly contains insulin-like receptor DAF-2 and forkhead transcription factor DAF-16 which is negatively regulated by DAF-2 [29]. After DAF-2 is inactive, DAF-16 nuclear is translocated from the cytoplasm to nuclei. Then, activation DAF-16 regulates stress response, life span, and metabolism. Since the activation of DAF-16 (mammalian homologue Foxo3) in C. elegans can extend lifespan, and resist stress and infection [30-32], we hypothesized that gastrodin might mediate neuroprotection by activation of DAF-16. We first determined the chemotaxis behavior of the daf-16 mutant. We found that knockdown of daf-16 resulted in reduced olfactory function (Fig. 3c). Then, we found that gastrodin could obviously increase content of dopamine in wild type animals $(P=0.0013<0.01, F=3.524, d f=4)$, and the dopamine in daf-16(mu86) mutant was reduced by LC-MS/MS method $(P=0.7565>0.05, F=1.089$, $d f=4$ ) (Additional file 2: Fig. S2). We then treated the daf-16 mutant with gastrodin. If gastrodin mediated neuroprotection through activation of DAF-16, then we would expect that gastrodin treatment would not be able to improve the deficient chemotaxis behavior of the daf-16 mutant. Consistent with this model, no response to gastrodin was observed $(P=0.8514>0.05$, $F=1.376, d f=4$; Fig. 3c).

It is known that DAF-16 activation is controlled by nuclear accumulation [33-35]. We next asked if gastrodin could induce nuclear accumulation of DAF-16:: GFP. We found that gastrodin activated DAF-16 and induced DAF-16 nuclear translocation after $24 \mathrm{~h}$ treated with gastrodin $(P<0.0001, F=3.035, d f=18$; Fig. $3 d$, e). These results indicated that gastrodin functions via DAF-16 to counter the symptoms of Parkinson's disease. 


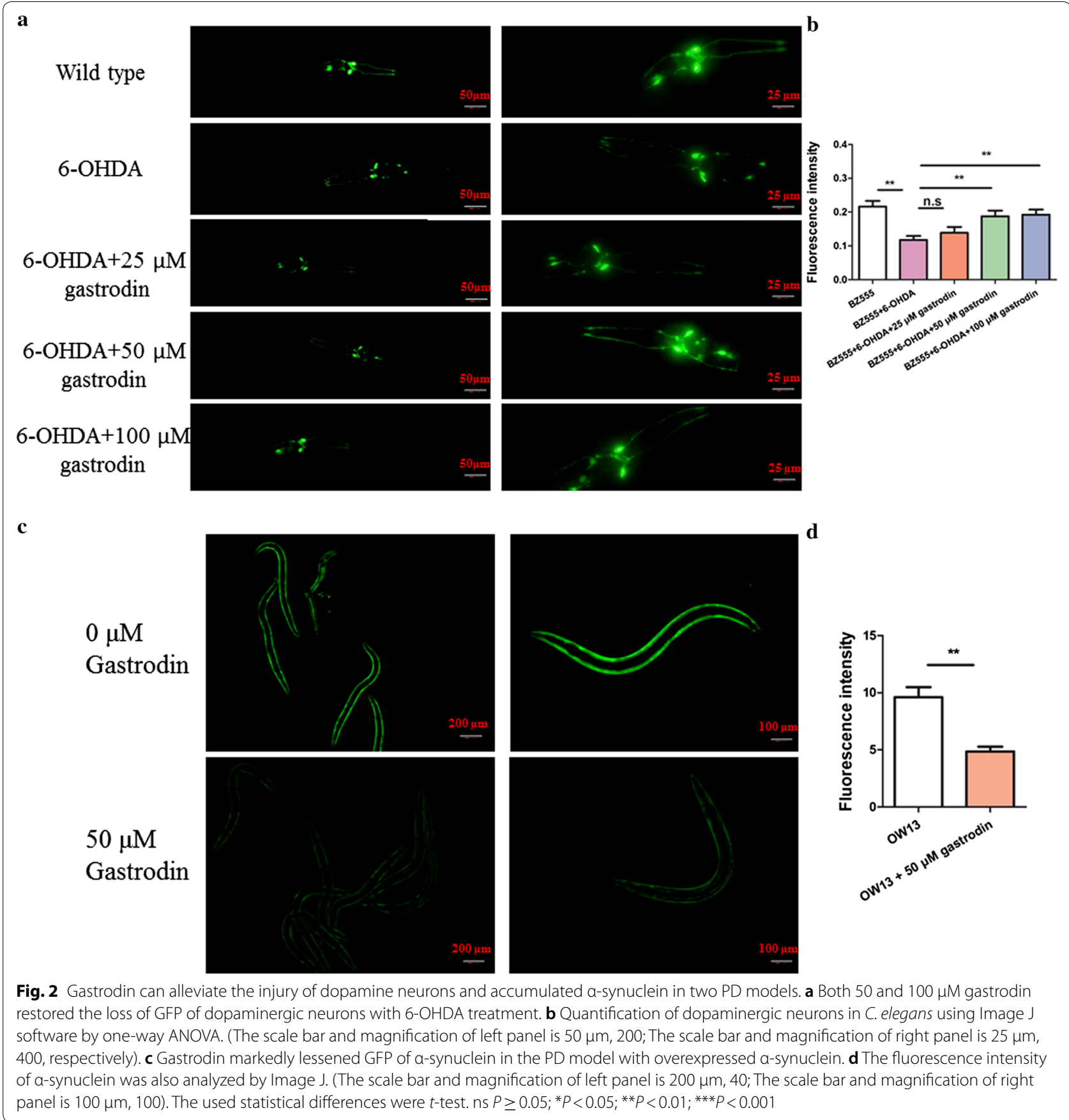

We next asked whether DAF-2 acted upstream of DAF-16 to confer anti-Parkinson properties. Compared with the wild type strain (N2), the daf-2 mutant exhibited significantly increased chemotaxis $(P=0.0078<0.01$, $F=2.351, d f=4$; Fig. 3f). Moreover, the double mutant daf-2(e1370); daf-16(mgDf47) obviously rescued the chemotaxis index to the level of wild type animals $(P=0.0327<0.05, F=1.856, d f=4 ;$ Fig. 3f $)$. Together, our data preliminarily confirmed that gastrodin could act to protect from Parkinson's disease via the DAF-2/DAF-16 signaling pathway.

\section{DAF-2 is required for neuroprotective effect of dopamine neuron in PD}

The above data suggest a role of the insulin-like pathway in PD, and we next used a genetic method to test 

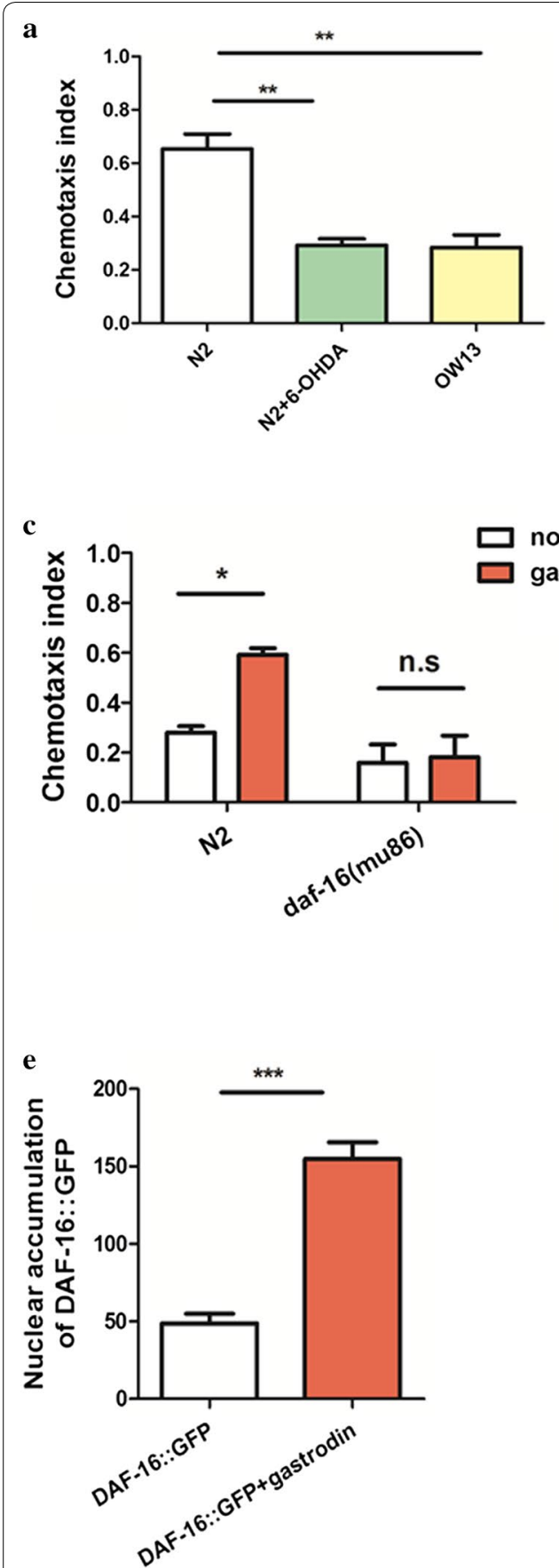
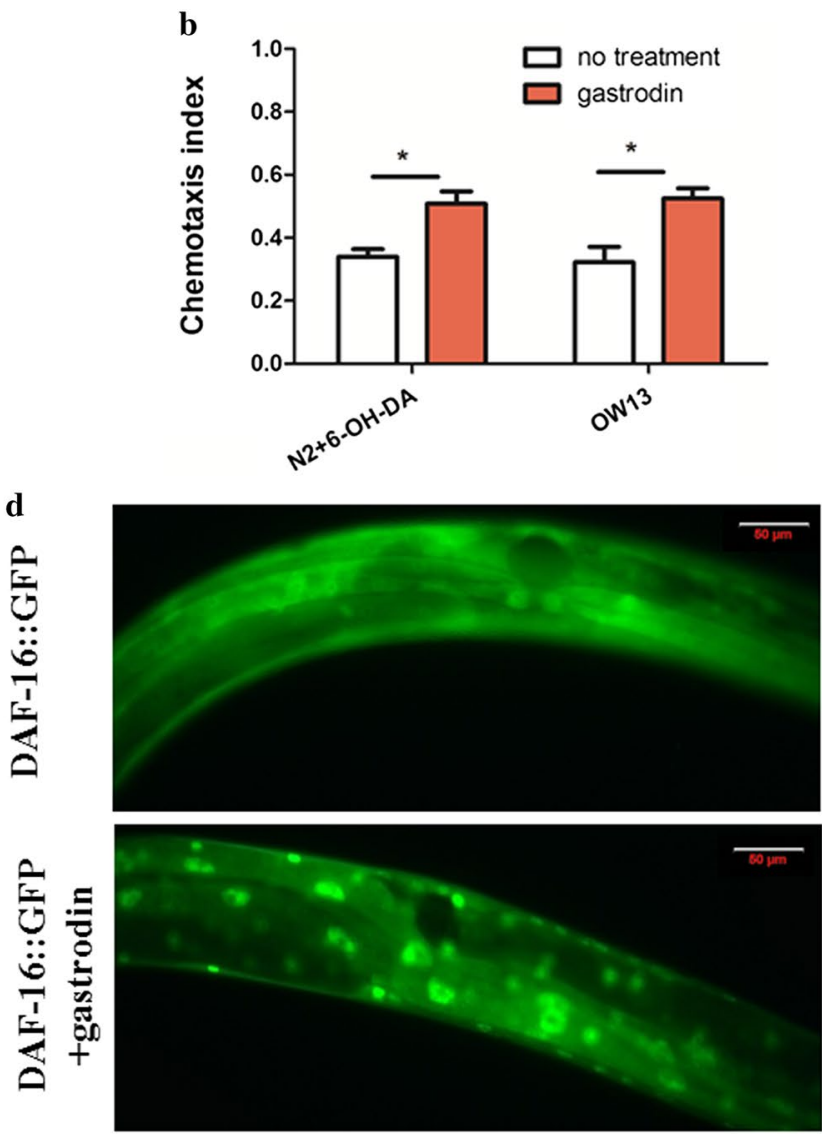

$\mathbf{f}$

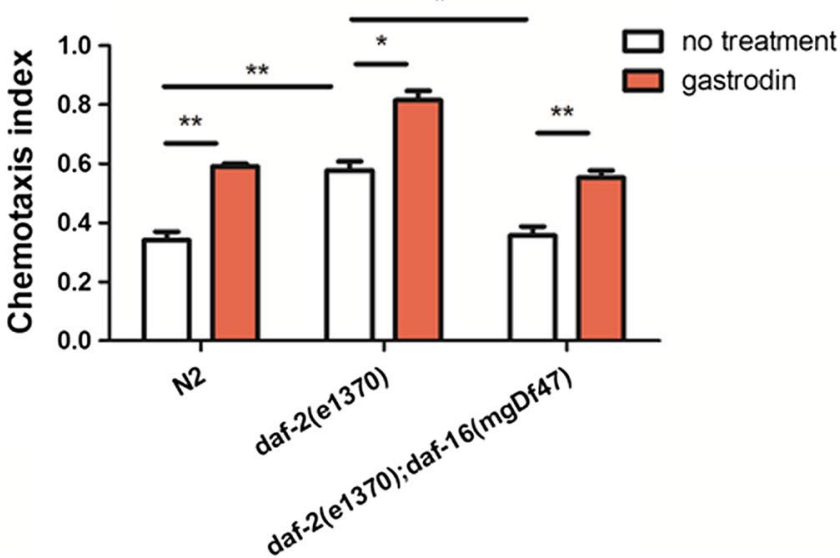

Fig. 3 Gastrodin rescues chemotaxis behavior by DAF-2/DAF-16. a In the two PD models, the chemotaxis behavior of worms was increased after treatment with gastrodin. $\mathbf{b}$ Gastrodin obviously increased chemotaxis behavior in two PD models. cThe mutant daf-16(mu86) shows decreased chemotaxis compared to wild type, and treatment of the mutant with gastrodin did not rescue the behavior. $\mathbf{d}$ Gastrodin increased the nuclear accumulation of DAF-16::GFP. The scale bar and magnification of left panel is $50 \mu \mathrm{m}$, 200. e Quantification of DAF-16::GFP nuclear accumulation in no gastrodin and gastrodin conditions. $\mathbf{f}$ daf-2 mutation exhibited an increased chemotaxis index, and the double-mutants daf-2; daf- 16 restored the level to that of wild type animals. The statistical differences were analyzed using $t$-test method, ns $P \geq 0.05 ;{ }^{*} P<0.05 ;{ }^{* *} P<0.01 ;{ }^{* * *} P<0.001$ 
if insulin-like signaling could protect dopamine neurons from degeneration. To obtain the double mutant, we crossed BZ555 [egIs1 [dat-1p:: GFP]] with the daf-2 mutant. Then, it was found that the GFP signal corresponding to dopamine neurons in the daf-2(e1270) mutant was unchanged relative to the double mutant daf2(e1370); egIs1[dat-1p::GFP] $(P=0.1966>0.05, F=11.4$, $d f=4$; Fig. 4a, b). However, we observed a change in the level of GFP in response to treatment with 6-OHDA in wild type worms and daf-2 mutant worms. As shown in Fig. 4c, d, BZ555 animals showed decreased GFP expression in dopamine neurons, and the daf-2 mutant significantly resisted 6-OHDA treatment compared with wild type worms $(P=0.029<0.05, F=3.42, d f=4$; Fig. $4 \mathrm{c}$, d). When we crossed OW13 [grk-1(ok1239) X; pkIs2386 $I V]$ with the $d a f-2$ mutant, we found no change in the accumulation of $\alpha$-synuclein in the daf-2(e1270); grk1 (ok1239) X; pkIs2386 IV $(P=0.3464>0.05, F=2.29$, $d f=12$; Fig. 4e, f). Our results further suggest that DAF-2 only mediated the activity of dopamine neurons not $\alpha$-synuclein. All of the data indicate that gastrodin may alleviate dopamine neuron injury and $\alpha$-synuclein accumulation, and mediates neuroprotection of dopaminergic neurons by the DAF-2/DAF-16 insulin-like pathway in the PD model.

In conclusion, we found that gastrodin regulated the DAF-2/DAF-16 pathway to repair the injury of dopamine neurons but not to reduce $\alpha$-synuclein accumulation in PD model. Therefore, the decreased levels of $\alpha$-synuclein protein resulting from gastrodin treatment are likely mediated by other pathways in the PD model.

\section{Discussion}

Gastrodin has been extensively studied in the treatment of neurodegenerative disease, but its mechanism remains to be elucidated. Our results indicate that gastrodin may attenuate dopamine neuron injury and $\alpha$-synuclein accumulation, and mediates neuroprotection of dopaminergic neurons via the DAF-2/DAF-16 insulin-like pathway in the PD model. Gastrodin mediates oxidative stress, ERK1/2 and GSK-3 $\beta$ pathways, apoptosis, and p38 MAPK/Nrf2 pathway to protect dopamine neurons in several PD models [14-17]. But, up to now, there are little reports that gastrodin alleviate PD through interfering with insulin pathway. Numberous studies have highlighted that insulin signaling is involved in neurodegeneration [26-28]. Likewise in $C$. elegans PD model, insulin-IGF-1 receptor DAF-2 mutation eases $\alpha$-synuclein aggregation, resist more different stress [27]. Morris and co-workers demonstrate that insulin resistance could damage the dopaminergic neurons via increased iron accumulation [36]. It is reported that IGF-1/insulin-like signaling modulates dopamine neurodegeneration induced by $\alpha$-synuclein independent of DAF-16/FOXO in Drosophila and C. elegans models [37]. Our study also found that DAF-2 is a key gene in PD model treated with gastrodin, however, DAF-16 which is a transcription factor acts downstream of DAF-2 to resist injury of dopamine neurons in C. elegans PD model.

Gastrodin still has effect on other central nervous system disease, including epilepsy, Alzheimer's disease, and even affective disorders, cognitive impairment. Gastrodin suppresses protein MAPK-associated inflammatory response, apoptosis in epilepsy [38, 39]. Studies have shown that pretreatment of gastrodin could antiinflammatory, anti-oxidative, anti-apoptotic an so on for decreasing the production and aggregation of $A \beta$ against Alzheimer's disease [40-42]. Based on previous reports, DAF-2 refers to aging, glucose metabolism, toxic protein accumulation [26, 37]. And, gastrodin can modulate DAF-2/DAF-16 in our result. Hence, it is speculated that gastrodin may delay aging, improve metabolism and reduce proteotoxicity to prevent other neurodegenerative disease by insulin-like signaling.

Many patients with Parkinson's disease exhibit nonmotor symptoms of olfaction dysfunction, but olfaction assessment is not a clinical diagnosis. Ethanol preference and nonanol repulsion assay has been reported to be used as a chemotaxis behavior phenotypes in PD models of C. elegans [43, 44]. From our data, it was found that chemotaxis behavior to 2-heptanone was an indicator of olfaction dysfunction in the C. elegans PD model. However, chemotaxis behavior to benzaldehyde was unaffected in our assay (data not shown). The responses to 2-heptanone and benzaldehyde depend on AWC chemosensory neurons, but the downstream signaling of the two odorants is different, involving G-protein-coupled receptors (GPCRs) and $\mathrm{G}$ protein, respectively $[45,46]$. Hence, we suggest that dopamine neuron damage and $\alpha$-synuclein accumulation do not result in the olfactory dysfunction of AWC neurons. The chemotaxis assasy to 2-heptanone in PD model could be a damaged downstream signaling pathway of AWC chemosensory neurons. But, we advised that the chemotaxis behavior to 2-heptanone might be an indicator in PD model. In addition, after treatment with gastrodin, the chemotaxis index of the daf-2(e1370) mutant was still obviously higher than for animals without gastrodin as shown in Fig. 3f. Thus, gastrodin may act on an additional signal pathway to activate DAF-16 to protect against Parkinson's disease.

\section{Conclusions}

Here, we found that gastrodin regulated the DAF-2/DAF16 pathway to repair the injury of dopamine neurons but not to reduce $\alpha$-synuclein accumulation in the $C$. elegans 


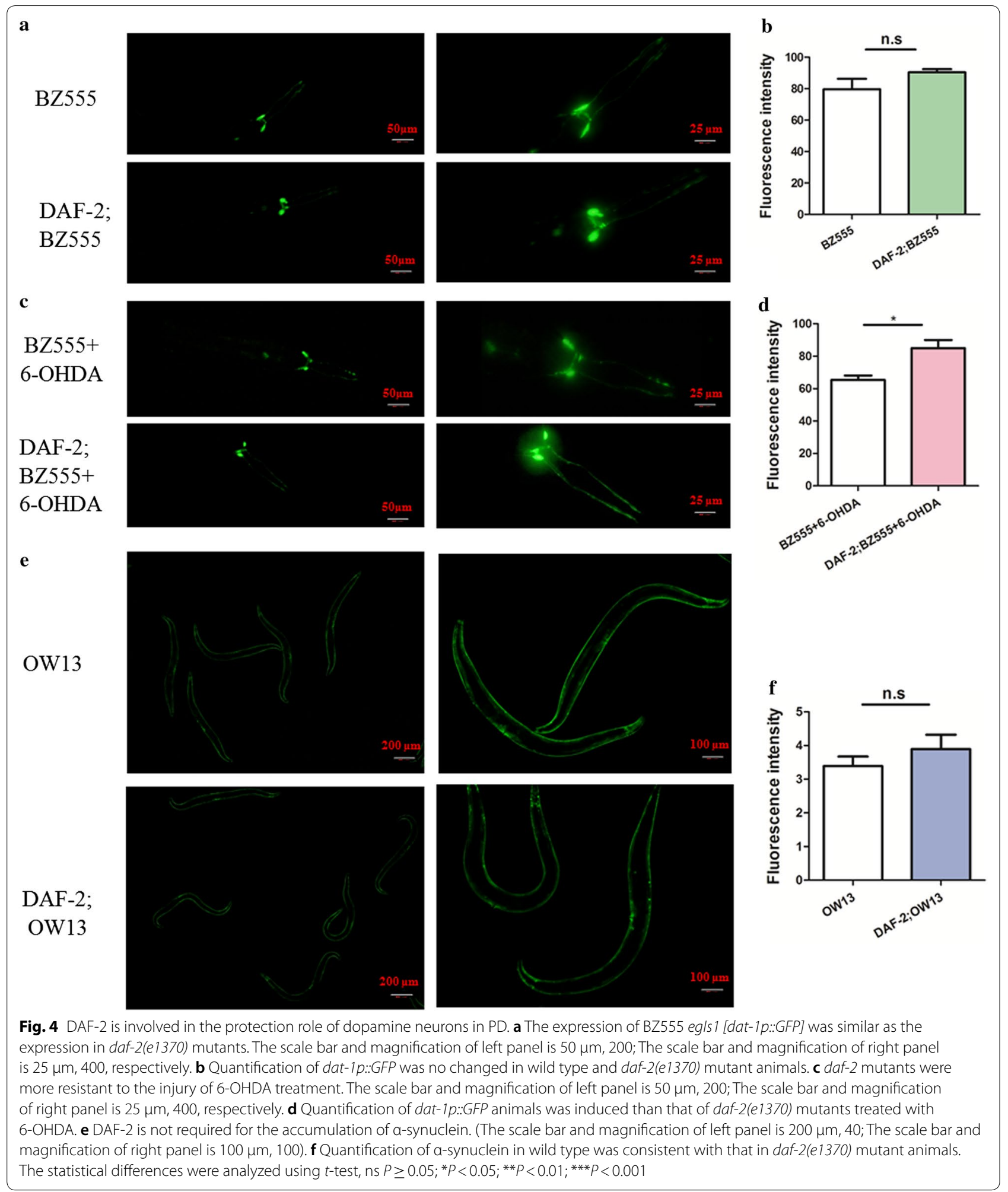


PD model. Therefore, the decreased levels of $\alpha$-synuclein protein resulting from gastrodin treatment are likely mediated by other pathways in the PD model.

\section{Methods}

\section{Strains of $C$. elegans}

The $C$. elegans strains used in this experiment were obtained from Caenorhabditis Genetics Center in Table 1. Nematodes were maintained and grown on nematode growth media (NGM) plates with Escherichia coli strain OP50 culture at $20{ }^{\circ} \mathrm{C}$ according to the standard protocol [47].

\section{Screening right concentration of gastrodin assay} Gastrodin was obtained from Sigma-Aldrich (SMB00313, USA). The gastrodin was dissolved with $\mathrm{dd}_{2} \mathrm{O}$ for $5 \mathrm{mM}$. Then, different volume gastrodin was diluted in NGM medium to different concentraions $(25,50,100$, $200 \mu \mathrm{M})$. The right concentration of gastrodin assay was evaluated by food clearance assay and pumping rate assay. The different concentrations $(0,25,50,100$, or $200 \mu \mathrm{M})$ of gastrodin was added in NGM or S medium and determined by monitoring the food clearance and the pumping rate.

In the food clearance assay, the same OD (optical density) of freshly incubated OP50 culture, about 35 L1 wild type worms and five different concentrations gastrodin were added to 96-well plates having $S$ medium. The plates containing $C$. elegans and OP50 culture were placed in $25^{\circ} \mathrm{C}$, then the OD595 $\mathrm{nm}$ of OP50 was determined by Microplate Reader daily.

Pumping rate was calculated by using microscopy, assessed on OP50/NGM plates. The pumping rate means the pharyngeal movement of worms. At the beginning of the test, thirty L4 worms were transferred onto each plate with a worm picker. After $24 \mathrm{~h}$, the pumping rate was determined for 10 individuals, located inside the bacterial lawn, by counting the pharynx grinder movements within a $30 \mathrm{~s}$ period.

\section{Behavior analysis}

In our assay, the olfactory dysfunction of PD model animals was assayed by measuring the chemotaxis response of animals to 2-heptanone (537683, SigmaAldrich, USA). Based on the reports by Bargmann, the chemotaxis index assay was performed as described previously in $10 \mathrm{~cm}$ culture plates containing $10 \mathrm{ml}$ of $1.6 \%$ agar [48]. There were two marks on the back at $0.5 \mathrm{~cm}$ edge of the assay plates symmetrically. Then, $1 \mu \mathrm{l} 10^{-1} 2$-heptanone (diluted in ethanol) was dropped in one mark, and $1 \mu \mathrm{l}$ control reagent ethanol was dropped in the other symmetrical mark. Then, the well-fed animals without bacteria after washed using M9 buffer were put in the center of plates. The numbers of worms at the two sides were counted respectively after $1 \mathrm{~h}$. The chemotaxis index was scored using the formula = worms numbers at 2-heptanone side worms numbers at control side/total animal number. The behavior assay had been repeated three times with 30-40 worms per assay.

\section{C. elegans model of PD and Gastrodin treatment}

We established PD model by 6-Hydroxydopamine (6-OHDA) according to Nass's report [49]. About 150 L3 stage worms were harvest, washed with M9 buffer, and added to a mixture including $10 \mathrm{mM}$ ascorbic acid and $50 \mathrm{mM}$ 6-OHDA. The worms were treated for $1 \mathrm{~h}$ at $24{ }^{\circ} \mathrm{C}$, mixed mildly every $15 \mathrm{~min}$, washed with $\mathrm{M} 9$ buffer. After the PD model was successfully established, the animals were divided into two groups including PD model group and gastrodin group. The animals of $\mathrm{PD}$ model group were laid in OP50/NGM medium addition with no gastrodin for $24 \mathrm{~h}$, then treated with floxuridine (purchased from Sigma, F0503) in $22^{\circ} \mathrm{C}$; The animals of gastrodin group were laid in OP50/NGM medium addition with gastrodin for $24 \mathrm{~h}$, then treated with gastrodin and floxuridine in $22{ }^{\circ} \mathrm{C}$. Both of the control group and gastrodin group were observed and further test

\section{Table 1 The strains used in the study}

\begin{tabular}{lll}
\hline Name & Genotype & Description \\
\hline N2 & C. elegans wild isolate & C. elegans var Bristol \\
BZ555 & egls1 & egls1 [dat-1 p::GFP] \\
OW13 & grk-1(ok1239)X; pkls2386 IV & pkls2386[unc-54p::alpha-synnuclein::YFP + unc-119(+)] \\
CB1370 & daf-2(e1370) III & daf-2(e1370) III \\
GR1309 & daf-16(mgDf47)l; daf-2(e1370) III & mgDf7 completely suppresses daf-c phenotype of daf-2. mgDf47 \\
& & deletes approximately 8 kb of the daf-16 gene beginning after \\
exon 4
\end{tabular}


after $72 \mathrm{~h}$ in Fig. 1a. Every experiment was performed at least three independent replicates.

\section{Crossing worms}

We obtained double mutants by crossing techniques [50]. The crosses were done by 6-10 males and 2 L4 stage hermaphrodites, and then the homozygous progeny was screened by Polymerase Chain Reaction (PCR) assay. After F1 produced, if there were male and female worms with fluorescent marker, the F1 generation is crossed successfully. Further, we also cloned a 602 bp daf-2 fragment for verify crossed $\mathrm{F} 1$ generation using primers CATCAA GATCCAGTGCTTCTG and GACGGACTGCA

-ATTTTTCG. Then, we allow F1 generation to selfcross for screening the homozygous progeny. We still cloned DAF-2 gene by PCR. Due to only one base mutation in daf-2(e1370) mutant, we sequenced the double stranded PCR product to obtain homozygous crossed worms.

\section{Quantifying of fluorescence}

The two PD models were treated with or without gastrodin as the above methods. The fluorescence of dopaminergic neurons and $\alpha$-synuclein expression was observed using Olympus BX51 fluorescence microscopy (Tokyo, Japan) after $24 \mathrm{~h}, 48 \mathrm{~h}, 72 \mathrm{~h}$. The fluorescence intensity of dopaminergic neurons and $\alpha$-synuclein was obtained by Image J software. The mean fluorescence intensity was measured at least three animals.

\section{DAF-16:: GFP expression analysis}

DAF-16::GFP worms in L3 stage were treated with gastrodin or no gastrodin in NGM plates having floxuridine. After $24 \mathrm{~h}, 48 \mathrm{~h}, 72 \mathrm{~h}$, the distribution of DAF-16::GFP was observed using Olympus BX51 fluorescence microscopy (Tokyo, Japan). The nuclear location of DAF$16:$ GFP was counted by the accumulation of GFP in the nuclear.

\section{Determination of dopamine content}

The content of dopamine were performed by a highperformance liquid chromatography system (LC-30A, Shimadzu, Japan) coupled with tandem mass spectrometer (API 3200, AB Sciex, USA).The synchronized worms were washed with M9 buffer to remove bacteria, harvested in $500 \mu \mathrm{l} 0.2 \mathrm{M} \mathrm{HClO}_{4}$, and then sonicated and centrifuged at $12000 \mathrm{rpm}$ for $15 \mathrm{~min}$. The supernatants were prepared for quantification of dopamine.

\section{Statistical analysis}

All data were analyzed using GraphPad Prism. no significant (ns) $P>0.05 ;{ }^{*} P<0.05,{ }^{* *} P<0.01$, ${ }^{* * *} P<0.001$. Three independent trails were performed.

\section{Additional files}

\begin{abstract}
Additional file 1: Fig. S1. The dopamine neurons of worms treated with gastrodin is no changed. The fluorescence intensity of BZ555 with gastrodin was similar to that of worms without gastrodin using $t$ test, ns $P \geq 0.05$, ${ }^{*} P<0.05,{ }^{* *} P<0.01$, ${ }^{* * *} P<0.001$.

Additional file 2: Fig. S2. DAF-16 increased the content of dopamine by LC-MS/MS. (A) The content of dopamine in wild type worms and daf16(mu86) mutant treated with gastrodin using LC-MS/MS. (B) Quantification of wild type worms and daf-16(mu86) mutant having gastrodin or no gastrodin by $t$-test, ns $P \geq 0.05,{ }^{*} P<0.05$, ${ }^{* *} P<0.01$, ${ }^{* * *} P<0.001$.
\end{abstract}

\section{Abbreviations}

PD: Parkinson's disease; TCM: traditional Chinese medicine; C. elegans: Caenorhabditis elegans; 6-OHDA: 6-Hydroxydopamine; MAPK: mitogen-activated protein kinases; PCR: Polymerase Chain Reaction; NGM: nematode growth media; OD: optical density; GFP: green fluorescent protein; GPCRs: G-proteincoupled receptors.

\section{Acknowledgements}

We thank Professor Xiaowei Huang (State Key Lab for Conservation and Utilization of Bio-Resources, Yunnan University) for wild type and three mutant worms.

\section{Authors' contributions}

$J Y$ and ZY contributed to conception and design, made experiment, analysis and interpretation of data in the manuscript; ZL was implied in collecting behavioural data; JY and NZ revised the manuscript; XC design and supervised this study. All authors read and approved the final manuscript.

\section{Funding}

The design of this study, writing the manuscript and data collection was supported by the National Natural Science Foundation of China under Grant [No. 31860274]; YNCUB [No. 2017KF009]. The collection, analysis, and interpretation of data was supported by Yunnan Provincial Science and Technology Department [Nos. 2017FE468(-155), CXTD201703, 2017IB011].

\section{Availability of data and materials}

The datasets used or analysed during the current study are available from the corresponding author on reasonable request.

\section{Ethics approval and consent to participate}

Not applicable.

\section{Consent for publication}

Not applicable.

\section{Competing interests}

There is no conflict of interest in this submission. I declared on behalf of my co-authors that the work described here is original research that has not been published previously, and meet the criteria for authorship. All of the authors approved the manuscript for publication

\section{Author details}

${ }^{1}$ The Second Affiliated Hospital of Kunming Medical University, 374 Dian Mian Road, Kunming 650101, Yunnan, China. ${ }^{2}$ Faculty of Basic Medicine, Yunnan University of Chinese Medicine, Kunming 650500, Yunnan, China.

Received: 10 February 2019 Accepted: 10 June 2019

Published online: 17 June 2019 


\section{References}

1. Rocca WA. The future burden of Parkinson's disease. Mov Disord. 2018:33(1):8-9.

2. World Health organization. Ageing and health. 2015. http://www.who. int/mediacentre/factsheets/fs404/en/. Accessed 11 Jan 2015.

3. Spillantini MG, Schmidt ML, Lee VMY, Trojanowski JQ, Jakes R, Goedert M. a-Synuclein in Lewy bodies. Nature. 1997;388(6645):839-40.

4. And CWO, Tatton WG. Etiology and pathogenesis of Parkinson's disease. Annu Rev Neurosci. 2009:22(6):123-44.

5. Grünblatt E. Parkinson's disease: molecular risk factors. Parkinsonism Relat Disord. 2012;18(Suppl 1):S45-8.

6. Henry H, Singleton AB. The genetics and neuropathology of Parkinson's disease. Acta Neuropathol. 2012;124(3):325-38.

7. Obeso JA, Olanow CW, Nutt JG. Levodopa motor complications in Parkinson's disease. Trends Neurosci. 2000;23(10 Suppl):S2-7.

8. Jin M, He Q, Zhang S, Cui Y, Han L, Liu K. Gastrodin suppresses pentylenetetrazole-induced seizures progression by modulating oxidative stress in Zebrafish. Neurochem Res. 2018;43(4):904

9. Ji-Nan D, Yi Z, Lian-Mei Z, Yue-Min L, Wei Z, Li-Gong B, Qing-Long A, YiDan L, Jun S, Di L. Gastrodin inhibits expression of inducible NO synthase, cyclooxygenase-2 and proinflammatory cytokines in cultured LPSstimulated microglia via MAPK pathways. PLoS ONE. 2011;6(7):e21891.

10. Sun W, Miao B, Wang XC, Duan JH, Ye X, Han WJ, Wang WT, Luo C, Hu SJ. Gastrodin inhibits allodynia and hyperalgesia in painful diabetic neuropathy rats by decreasing excitability of nociceptive primary sensory neurons. PLoS ONE. 2012;7(6):e39647.

11. Wang Y, Wu Z, Liu X, Fu Q. Gastrodin ameliorates Parkinson's disease by downregulating connexin 43. Mol Med Rep. 2013;8(2):585.

12. Hsieh MT, Wu CR, Chen CF. Gastrodin and p-hydroxybenzyl alcohol facilitate memory consolidation and retrieval, but not acquisition, on the passive avoidance task in rats. J Ethnopharmacol. 1997:56(1):45-54.

13. Lei-Chwen L, Yen-Fei C, Wen-Chuan L, Yu-Tse W, Tung-Hu T. Pharmacokinetics of gastrodin and its metabolite $p$-hydroxybenzyl alcohol in rat blood, brain and bile by microdialysis coupled to LC-MS/MS. J Pharm Biomed Anal. 2008;48(3):909-17.

14. Haddadi R, Poursina M, Zeraati F, Nadi F. Gastrodin microinjection suppresses 6-OHDA-induced motor impairments in parkinsonian rats: insights into oxidative balance and microglial activation in SNc. Inflammopharmacology. 2018;26(5):1305-16

15. Li Q, Niu C, Zhang X, Dong M. Gastrodin and isorhynchophylline synergistically inhibit MPP+-induced oxidative stress in SH-SY5Y cells by targeting ERK1/2 and GSK-3i ${ }^{2}$ pathways: involvement of Nrf2 Nuclear Translocation. ACS Chem Neurosci. 2017;9(3):482-93.

16. Kumar H, Kim I-S, More SV, Kim B-W, Bahk Y-Y, Choi D-K. Gastrodin protects apoptotic dopaminergic neurons in a toxin-induced Parkinson's disease model. Evid Based Complement Altern Med 2013; 2013:514095.

17. Jiang G, Hu Y, Liu L, Cai J, Peng C, Li Q. Gastrodin protects against MPP(+)induced oxidative stress by up regulates heme oxygenase- 1 expression through p38 MAPK/Nrf2 pathway in human dopaminergic cells. Neurochem Int. 2014;75(9):79.

18. Cindy V, Hemant V, Nicola W, Bates EA, Stockwell BR, Hart AC. Identification of potential therapeutic drugs for huntington's disease using Caenorhabditis elegans. PLoS ONE. 2007;2(6):e504.

19. Chen YM, Liu SP, Lin HL, Chan MC, Chen YC, Huang YL, Tsai MC, Fu RH. Irisflorentin improves a-synuclein accumulation and attenuates 6-OHDAinduced dopaminergic neuron degeneration, implication for Parkinson's disease therapy. BioMedicine. 2015;5(1):4.

20. Trojanowski NF, Raizen DM, Fangyen C. Pharyngeal pumping in Caenorhabditis elegans depends on tonic and phasic signaling from the nervous system. Sci Rep. 2016;6:22940.

21. Doty RL, Golbe LI, Mckeown DA, Stern MB, Lehrach CM, Crawford D. Olfactory testing differentiates between progressive supranuclear palsy and idiopathic Parkinson's disease. Neurology. 1993;43(5):962-5.

22. Berendse HW, Booij J, Francot CM, Bergmans PL, Hijman R, Stoof JC, Wolters EC. Subclinical dopaminergic dysfunction in asymptomatic Parkinson's disease patients' relatives with a decreased sense of smell. Ann Neurol. 2001;50(1):34-41.

23. Bsc EH, Uylings HBM, PhD PVHM. A 100\% increase of dopaminergic cells in the olfactory bulb may explain hyposmia in Parkinson's disease. Mov Disord. 2004;19(6):687-92.
24. Doty RL. Olfactory dysfunction in Parkinson's disease. Focus on Parkinsons Disease. 2014;24(1):38-43.

25. Maulik M, Mitra S, Bult-Ito A, Taylor BE, Vayndorf EM. Behavioral phenotyping and pathological indicators of Parkinson's disease in C. elegans models. Front Genet. 2017:8:77.

26. Cohen E, Dillin A. The insulin paradox: aging, proteotoxicity and neurodegeneration. Nat Rev Neurosci. 2008;9(10):759-67.

27. Cooper JF, Dues DJ, Spielbauer KK, Machiela E, Senchuk MM, Raamsdonk JMV. Delaying aging is neuroprotective in Parkinson's disease: a genetic analysis in C. elegans models. NPJ Parkinsons Dis. 2015;1(1):15022.

28. Fatima S, Haque R, Jadiya P, Shamsuzzama, Kumar L, Nazir A. Ida-1, the Caenorhabditis elegans orthologue of mammalian diabetes autoantigen IA-2, potentially acts as a common modulator between Parkinson's disease and diabetes: role of Daf-2/Daf-16 insulin like signalling pathway. Plos One. 2014;9(12):e113986.

29. Millet AC, Ewbank JJ. Immunity in Caenorhabditis elegans. Curr Opin Immunol. 2004;16(1):4-9.

30. Lee SS, Kennedy S, Tolonen AC, Ruvkun G. DAF-16 target genes that control C. elegans life-span and metabolism. Science. 2003;300(5619):644.

31. Chávez V, Mohrishiomi A, Maadani A, Vega LA, Garsin DA. Oxidative stress enzymes are required for DAF-16-mediated immunity due to generation of reactive oxygen species by Caenorhabditis elegans. Genetics. 2007;176(3):1567

32. Zou CG, Tu Q, Niu J, Ji XL, Zhang KQ. The DAF-16/FOXO transcription factor functions as a regulator of epidermal innate immunity. PLoS Pathog. 2013;9(10):e1003660.

33. Henderson ST, Johnson TE. daf-16 integrates developmental and environmental inputs to mediate aging in the nematode Caenorhabditis elegans. Curr Biol. 2001;11(24):1975-80.

34. Lin K, Hsin H, Libina N, Kenyon C. Regulation of the Caenorhabditis elegans longevity protein DAF-16 by insulin/IGF-1 and germline signaling. Nat Genet. 2001;28(2):139-45.

35. Lee RYN, Hench J, Ruvkun G. Regulation of C. elegans DAF-16 and its human ortholog FKHRL1 by the daf-2 insulin-like signaling pathway. Curr Biol. 2001;11(24):1950-7.

36. Morris J, Bomhoff G, Gorres B, Davis V, Kim J, Lee P-P, Brooks W, Gerhardt $\mathrm{G}$, Geiger P, Stanford J. Insulin resistance impairs nigrostriatal dopamine function. Exp Neurol. 2011:231(1):171-80.

37. Knight A, Yan X, Hamamichi S, Ajjuri R, Mazzulli J, Zhang M, Daigle JG, Zhang S, Borom A, Roberts L. The glycolytic enzyme, GPI, is a functionally conserved modifier of dopaminergic neurodegeneration in Parkinson's models. Cell Metab. 2014:20(1):145-57.

38. Chen L, Liu X, Wang H, Qu M. Gastrodin attenuates pentylenetetrazoleinduced seizures by modulating the mitogen-activated protein kinaseassociated inflammatory responses in mice. Neurosci Bull. 2017;33(3):1-9.

39. Bian L, Bi X, Ai Q, Guo J, Dong S, Xu J, Zhong L, Di L. Effects of gastrodin on apoptotic factors of cerebral cortex neuron in epileptic rats. Chin J Neuroanat. 2016;32:37-43.

40. Zhao X, Zou Y, Xu H, Fan L, Guo H, Li X, Li G, Zhang X, Dong M. Gastrodin protect primary cultured rat hippocampal neurons against amyloidbeta peptide-induced neurotoxicity via ERK1/2-Nrf2 pathway. Brain Res. 2012;1482:13-21.

41. Hu Y, Li C, Shen W. Gastrodin alleviates memory deficits and reduces neuropathology in a mouse model of Alzheimer's disease. Neuropathology. 2015;34(4):370-7.

42. Geum-Hwa L, Hyung-Ryong K, Sang-Yong H, Bidur B, Do-Sung K, Min-Gul K, Byung-Ok S, Sun-Young K, Kyu-Sik J, Bo-Hee L. Gastrodia elata Blume and its pure compounds protect BV-2 microglial-derived cell lines against $\beta$-amyloid: the involvement of GRP78 and CHOP. Biol Res. 2012;45(4):403.

43. Lee J, Jee C, Mcintire SL. Ethanol preference in C. elegans. Genes Brain Behav. 2010;8(6):578-85.

44. Sashidhara KV, Modukuri RK, Jadiya P, Rao KB, Sharma T, Haque R, Singh DK, Banerjee D, Siddiqi MI, Nazir A. Discovery of 3-arylcoumarin-tetracyclic tacrine hybrids as multifunctional agents against Parkinson's disease. ACS Med Chem Lett. 2014;5(10):1099-103.

45. Zhang C, Yan J, Chen Y, Chen C, Zhang K, Huang X. The olfactory signal transduction for attractive odorants in Caenorhabditis elegans. Biotechnol Adv. 2014;32(2):290.

46. Zhang C, Zhao N, Chen Y, Zhang D, Yan J, Zou W, Zhang K, Huang X. The signaling pathway of $C$ elegans mediates chemotaxis response to the 
attractant 2-heptanone in a'Trojan Horse'- like pathogenesis. J Biol Chem. 2016;291(45):23618-27.

47. Brenner S. The genetics of Caenorhabditis elegans. Genetics. 1974;77(1):71.

48. Bargmann $\mathrm{Cl}$, Horvitz HR. Chemosensory neurons with overlapping functions direct chemotaxis to multiple chemicals in C. elegans. Neuron. 1991;7(5):729-42.

49. Richard N, Hall DH, Miller DM, Blakely RD. Neurotoxin-induced degeneration of dopamine neurons in Caenorhabditis elegans. Proc Natl Acad Sci USA. 2002:99(5):3264-9.
50. Friedman DB, Johnson TE. A mutation in the age-1 gene in Caenorhabditis elegans lengthens life and reduces hermaphrodite fertility. Genetics. 1988;118(1):75-86.

\section{Publisher's Note}

Springer Nature remains neutral with regard to jurisdictional claims in published maps and institutional affiliations.
Ready to submit your research? Choose BMC and benefit from:

- fast, convenient online submission

- thorough peer review by experienced researchers in your field

- rapid publication on acceptance

- support for research data, including large and complex data types

- gold Open Access which fosters wider collaboration and increased citations

- maximum visibility for your research: over $100 \mathrm{M}$ website views per year

At BMC, research is always in progress.

Learn more biomedcentral.com/submissions 